\title{
ENT trainee papers presented at the ENT Scotland Winter Meeting, 20 November 2015, Sterling, Scotland, UK
}

\begin{abstract}
A review of the effect of the new consultant-led ENT service in Greater Glasgow and Clyde on emergency admission care
\end{abstract}

H Rooney, O Hilmi

Glasgow Royal Infirmary

Aim

To assess if the new consultant-led service introduced to adult ENT services in Greater Glasgow and Clyde in February 2015 has led to improved patient care, using the surrogate markers of number of emergency admissions and average length of stay of emergency admissions.

\section{Methods}

The number of emergency admissions and average length of stay of emergency admissions were analysed from the time of the introduction of the consultant-led service for 28 weeks and compared with the previous 5 years for a comparable 28 weeks.

\section{Results}

The numbers of emergency admissions were not affected. The average length of stay has been reduced, although this was only deemed significant in four of the five preceding years.

\section{Conclusion}

The introduction of a consultant-led service has not impacted the number of emergency admissions, but may have significantly reduced the average length of stay. A longer time analysis is required to confirm this finding.

Treatments for otitis externa, from 1809 to 2015: a study of historical texts

E Dirks, E Stapleton, M Shanks

University Hospital Crosshouse, Kilmarnock

\section{Introduction}

There is a paucity of high-level evidence for otitis externa treatments, and a current worldwide focus on antibiotic resistance. Current guidelines recommend topical treatments, yet general practitioners give systemic antibiotics to 70 per cent of uncomplicated otitis externa patients.

Aim

To study historical trends in the treatment of otitis externa, before, during and after antibiotic development.

\section{Methods}

Historical texts were studied, including The Surgeon's VadeMecum (1809), Diseases of the Throat, Nose, and Ear
(Porter, 1919) and Logan Turner's Diseases of the Nose, Throat and Ear (editions I-XI; 1924-2015).

Results

Historical treatments for otitis externa included: warm water (1809); cocaine in glycerine, linear incisions and hydrogen peroxide (1919); 10 per cent sulpharsphenamine, icthymol and glycerin (1936); generous amounts of systemic sulphonamides (1952); topical antibiotics (1961); and night sedation and cotton gloves (1982).

\section{Conclusions}

Historical treatments were rarely evidence-based, and frequently fell from favour because they were unsafe. Randomised, controlled trials are required to clarify the efficacy of safe non-antibiotic treatments.

A prospective cohort study of adults receiving Gardasil vaccine for recurrent respiratory papillomatosis of the larynx: preliminary report

R Hurley, J Montgomery, A Harrison, F B MacGregor, M A Buchanan, K MacKenzie

Glasgow Royal Infirmary

\section{Aim}

Recurrent respiratory papillomatosis is caused by human papilloma virus (HPV) strains 6 and 11 . Gardasil ${ }^{\circledR}$ is a vaccine active against HPV strains 6, 11, 16 and 18. This study aimed to observe any change in disease burden in patients with recurrent respiratory papillomatosis, before and after vaccination.

\section{Methods}

A prospective cohort of patients was observed for one year. Vaccinated patients formed the treatment group. Unvaccinated patients formed the non-treatment group. Primary outcome was the time between surgical interventions.

Results

Twenty-eight patients were identified. In the treatment group $(n=12), 42$ per cent of patients had not required further surgical intervention in the nine months following vaccination. In the non-treatment group $(n=16), 38$ per cent of non-vaccinated patients had not required further surgical intervention; hence, these findings were similar to those for the vaccinated patients $(p=1)$.

\section{Conclusion}

Patients receiving vaccination have a similar chance of disease remission in the short-term as those who are not vaccinated. Gardasil for recurrent respiratory papillomatosis, at present, appears to have no advantage over not vaccinating. 
Perceived importance of tonsillectomy outcomes: prospective analysis of a regional population

P Murnane, S Benzie, E M Shanks

University Hospital Crosshouse, Kilmarnock

\section{Problem}

Common indications for tonsillectomy in children are recurrent tonsillitis and sleep-disordered breathing. Extensive research exists on the potential benefits of tonsillectomy, but not on parental expectations.

\section{Aims}

To assess whether the expectations of parents of children undergoing tonsillectomy correspond to those of ENT surgeons, and to ascertain if there is the potential to improve communication with parents and patient education.

\section{Methods}

A prospective questionnaire study was conducted to assess the perceived importance of potential tonsillectomy outcomes. Parents' views were compared with those of ENT surgeons and analysed according to indication. Compliance with Scottish Intercollegiate Guidelines Network (SIGN) guidelines was assessed.

\section{Results}

Sixty-one patients were included in the study. Parents' and surgeons' expectations correlated well, but 'fewer sore throats' was more valued by parents, while surgeons attached more importance to 'fewer nocturnal breathing problems'. The SIGN criteria were met by 71.4 per cent of patients with tonsillitis and by 67.5 per cent of patients with concomitant sleep-disordered breathing.

\section{Conclusions}

Better understanding of parental expectations should allow improved pre-operative counselling tailored to the tonsillectomy indication. Improved adherence to SIGN guidelines is needed, although sleep-disordered breathing is not addressed by these.

Surgical management of nasal stenosis following chemoradiation for nasopharyngeal carcinoma

V Wilmot, I Hathorn

St John's Hospital, Edinburgh

\section{Introduction}

Nasal stenosis is a rare but troublesome consequence of chemoradiation for nasopharyngeal carcinoma (NPC) patients. It has a significant impact on quality of life and disease surveillance.

\section{Aim}

To present a surgical technique to manage post-chemoradiation nasal stenosis, with three supporting cases.

\section{Methods}

Three patients underwent endoscopic division of adhesions, posterior septectomy, posterior inferior turbinectomy and opening of posterior choanae bilaterally, performed by a single consultant rhinologist using image guidance. No splints were required. Regular saline rinses were used postoperatively.
Results

In all cases, the nasal stenosis was successfully addressed, with no intra-operative complications. At follow up $(2-13$ months post-operatively), all patients reported improvement in obstructive symptoms, as supported by improved Glasgow Benefit Inventory scores. There was no re-stenosis and disease surveillance was possible. One palliative patient, operated on for symptom relief, passed away two months postoperatively.

\section{Conclusions}

We describe a simple, effective endoscopic technique to improve nasal obstruction and allow disease surveillance in nasal stenosis cases following chemoradiation for NPC.

Speech and language led Throat Therapy Clinic: two years' experience

C Mamais, M Singer, P Asimakopoulos, M Bannister, K Ah-See

Aberdeen Royal Infirmary

\section{Introduction}

We designed a new service where an experienced speech and language therapist would see selected patients referred to our ENT services with dysphonia or globus sensation directly.

\section{Methodology}

Patients without any additional risk factors are vetted by our head and neck consultant and seen in the Throat Therapy Clinic. The laryngoscopy findings are recorded and reviewed at a later stage by the consultant.

\section{Results}

From 6 December 2012 to 2 July 2015, there were 500 appointments for 455 unique patients; 413 of the patients attended. The commonest reason for referral was globus sensation ( 64 per cent), followed by dysphonia ( 35 per cent). Of the patients, 325 were discharged with general advice, 31 required voice therapy, and 29 underwent investigations or were scheduled for surgery. The only one patient that was found to have a malignancy was originally referred routinely.

\section{Conclusion}

This new service is safe and effective, and it relies on adequate information being provided by the general practitioner.

\section{Trends in airway management in Scotland's} paediatric population

\section{K Lang, H Kubba}

Royal Hospital for Sick Children, Glasgow

\section{Introduction}

In the last 25 years, significant advances have occurred in paediatric airway management, as well as increasing subspecialisation and centralisation of neonatology, intensive care, and anaesthetic and surgical services. We present data on trends in paediatric airway surgery in Scotland.

\section{Methods}

Data were obtained from the Information Services Division of NHS Scotland for all airway procedures performed in patients aged 16 years or younger from 1989 to 2014 . We 
analysed open and endoscopic airway procedures by health board.

Results

A general upward trend in the number endoscopic procedures was reversed between 2003 and 2009, probably as a result of changes in the coding of awake fibre-optic laryngoscopy. This figure has now reached a high of 418 per year. The total number of open procedures increased from 31 in 1989 to 108 in 2014. The airway procedure ratio for the Greater Glasgow and Clyde Health Board to all other health boards was approximately 3:1.

\section{Conclusion}

There has been a rising demand for paediatric airway surgery in Scotland, particularly in the Scottish National Health Service in Glasgow.

Creating a novel staging system for salivary gland fine needle aspirates

J Rocke, K Ah-See

Aberdeen Royal Infirmary

Introduction

We created and successfully applied a cytopathological staging system for salivary gland fine needle aspiration (FNA). This increased the sensitivity of the technique, which will aid multidisciplinary communication and management of salivary swellings.

\section{Aims}

To create a salivary gland FNA staging system and demonstrate its efficacy.

\section{Methods}

Paired FNA and excision samples were identified after extraction of data from the electronic reporting system. Results were analysed comparatively to determine the accuracy of our staging technique compared to no staging.

Results

The staging system was easily applied to all salivary gland FNAs. Application of this system was shown to significantly increase diagnosis sensitivity $(p<0.05)$.

\section{Conclusions}

This staging system can be readily applied to salivary gland FNA cytology. This framework could be used to guide further research and aid multidisciplinary management of salivary gland lesions.

Is there a role for electrical stimulation in cases of significant facial palsy after revision pleomorphic adenoma surgery?

S Goldie, R Cole, S Dennis, J Sandeman, I Swain

Salisbury District Hospital

\section{Introduction}

Conflicting evidence exists regarding the benefits of electrical stimulation in facial palsy cases. Most studies describe facial electrical stimulation in the treatment of Bell's palsy. These studies demonstrate faster resolution, but report mixed findings with regards to its efficacy. Excision of pleomorphic adenomas using techniques such as total parotidectomy, superficial parotidectomy and extracapsular dissection carry significant risks of facial nerve damage, synkinesis and Frey's syndrome. These risks are far greater in revision cases. Ninety-five per cent of patients undergoing revision surgery will demonstrate a House-Brackmann facial nerve damage score of at least grade II, and 11.3 per cent will have a score of grade III or higher at one year after surgery.

\section{Methods and results}

We describe two case studies treated with electrical stimulation who suffered significant facial palsy after revision pleomorphic adenoma resection. Both patients recovered good facial function, as demonstrated by Sunnybrook facial grading scores of 54 and 64 that improved to 88 and 96 respectively after 24 weeks of treatment. Both resting facial symmetry and movement symmetry were noted to improve, and neither patient suffered complications of synkinesis or Frey's syndrome after treatment.

\section{Conclusions}

Facial stimulation may have a role in the treatment of significant facial palsy after revision pleomorphic adenoma surgery, although large-scale studies will be difficult to carry out because of the low incidence of cases. 